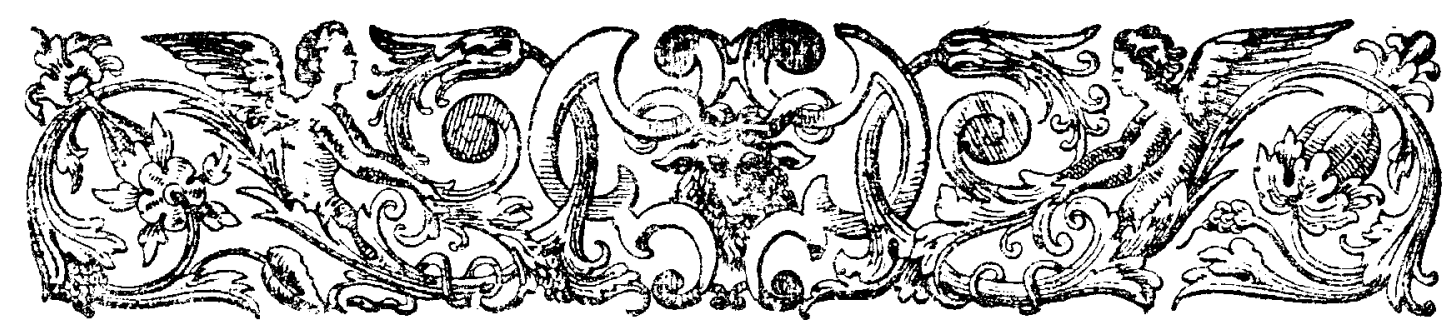

\title{
Ein Flügelaltar von Jacobus van Utrecht
}

\author{
VON † DR. W. NEUMANN, (Riga).
}

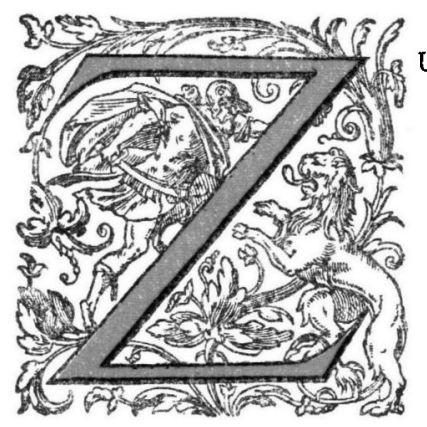

U den bis jetzt bekannten Werken, vier schönen Bildnissen von der Hand des Meisters Jacobus van UTRECht, oder wie er sich auf seinen Bildern zeichnete JACOBUS(CLAESSENS) TRAJECTENSIS, lässt sich heute ein Kleines Altarwerk, ein Hausaltar, hinzufügen, den erst kürzlich ein glücklicher Zufall, als von der Hand dieses Künstlers stammend, hat entdecken lassen. Er befindet sich im Besitz der Galerie des weil. rigaschen Ratsherren FrIEDrich WILHELM BREDERLo, die im Jahre 1905 mit den Sammlungen der Stadt Riga vereinigt in Fachkreisen durch ihre holländischen Kleinmeister und durch eine Anzahl guter Werke deutscher Meister aus der ersten Hälfte des r. Jahrhunderts bekannt geworden ist.

BREDERLO, seines Zeichens einer jener deutschen Grosskaufleute von altem Schlage, wie sie gelegentlich nur noch in Romanen geschildert werden, hatte seine Freude an der Kunst. Es war ihm eine Lust sich mit Bildern nach seinem Geschmack zu umgeben und so entstand allmählich die bei seinem Tode 20 I Nummern umfassende Galerie, die bei der Eröffnung des neuen Museumgebäudes als besondere Abteilung in ihm aufgestellt wurde. Auf seinen vielfachen Reisen, die ihn häufig nach Deutschland, Oesterreich, Frankreich, England und Schweden führten, erwarb BREDERLO seine Bilder im Kunsthandel, oder unmittelbar in den Ateliers der Künstler, doch fehlen leider mit geringen Ausnahmen, die sich durch Kombination ermitteln liessen, oder der Zufall brachte, Angaben über ihre Herkunft.

So kam um die Mitte des 19. Jahrhunderts auch der in Rede stehende kleine Flügelaltar in die Sammlung, ohne dass sich hätte ermitteln lassen, wo ihn BREDERLo erworben haben könnte. Das oben im Halbkreise schliessende Werk misst in der Höhe $0.77 \mathrm{~m}$. bei einer Breite von $0.6 \mathrm{r} 5 \mathrm{~m}$.

Auf den Aussenseiten der Flügel sieht man links den Evangelisten Johannes 
in rotem Mantel in blaugrünem Unterkleide, den Kelch segnend, aus dem sich die Schlange ringelt; rechts den Apostel Jacobus minor in blaugrünem Mantel und braunem Unterkleide mit Buch und Keule. Beide, schön gezeichnete, würdige Gestalten, stehen auf blumigem Rasen in einer von Bergen umschlossenen Landschaft. - Innen als Hauptbild die Madonna mit dem Christuskind; Kniestück. Sie trägt ein nerzverbrämtes, goldgesäumtes Unterkleid von leuchtend weinroter und ein Oberkleid von blaugrüner Farbe, dessen zurückgeschlagener rechter Aermel das hellblaue Futter sehen lässt. Das goldblonde Haar bedeckt ein duftiger Schleier. Sie stützt die rechte Brust mit der Hand und umfängt mit der Linken, den auf einem Goldbrokatkissen sitzenden nackten Jezusknaben, der den Genuss der Mutterbrust unterbrochen hat und mit einem an einem Faden flatternden Täubchen spielt. Hinter der Gruppe heben drei geflügelte Engelknaben einen goldgesäumten dunkellilafarbenen Vorhang empor, der zu beiden Seiten den Blick in eine von Höhenzügen begrenzte blühende Dorflandschaft freilässt: links ein herrschaftliches von Bäumen umstandenes Haus mit einem turmartigen Ausbau; im Vordergrunde ein Weiher mit Schwänen, an seinem Ufer ein Reiter. Rechts Wirtschaftsgebäude; vor ihnen eine Schafheerde mit einem Hirten. Auf dem Wege davor ein Sackträger. Im Hinter grunde sieht man vor einem Gebirgszuge dessen Spitzen von der Sonne beleuchtet sind, die mächtigen Rundtürme einer entfernten Stadt über dem Walde hervortreten.

Auf den Flügeln Donator und Donatrix anbetend in Halbfiguren. $\mathrm{Zu}$ ihren Haupten unter zierlich geschwungenem Laubwerk je zwei Wappen auf tiefschwarzem Grund. Der Donator, ein etwa vierzigjähriger Mann mit braunem, spärlichem Haar und kleinem Backenbart, in schwarzem pelzbesetztem Mantel und rotem Untergewand, (dessen beide Aermel leider übermalt sind) kniet mit gefalteten Händen vor einem grünbehangenen Altar, auf dem ein aufgeschlagenes Buch liegt. An seiner rechten Seite schaut ein kleines weisses Bologneserhündchen mit braunen Ohren zum Bilde hinaus. - Auf dem rechten Flügel, die ebenfalls vor einem grünbehan. genen Altar knieende Gattin des Stifters. Sie trägt ein weisses Kleid mit viereckigem Halsausschnitt und einen rotbraunen Sammetumhang um die Schultern. Die zum Gebet zusammengelegten Hände sind reich beringt, ruhen auf dem Altar und halten eine Kugel des auf ihm liegenden Rosenkranzes. Das blonde Haar verschwindet fast unter einer reichgestickten, mit Perlen besetzten Haube. Ein ähnlich geschmücktes Halsband, ein mit Edelsteinen besetztes an einer feinen Goldkette am Halse hängendes Brustkreuz, eine gleichfalls mit Edelsteinen reichbesetzte Agraffe am Schluss des Kleides und ein prächtiger Gürtel bilden den übrigen Schmuck der Dame. Die Wappen zu Häupten des Stifters; links: KERCKRING : aufgerichteter nach links schreitender schwarzer Löwe in Gold; Helmzier: Hundekopf auf langem Hals. Rechts: KASTORP gespaltener Schild; vorne halber schwarzer Adler in Gold; hinten 
viermal rot und schwarz geteilt. Helmzier: Adlerflug, rot und gold. Darnach ergibt sich, dass der Dargestellte (nach G. W. DITTMER: Genealogische und biographische Nachrichten über Lübeckische Familien aus älterer Zeit. Lübeck I 859) der Lübeckische Ratsherr HiNRICH KERCKRING ist, geb. 1479, Ratsherr seit I5 I 8, gest. I 540, Januar I 2., ein Sohn des Ratsherrn Johand Kerckring, Hinrichs SoHn (Ratsherr seit 1484, gest. 1516) und dessen Gattin TALECkE KaSTORP, Tochter des Bürgermeisters HINRICH KASTORP, gest. I488 1). Vermählt war HINRICH KERCKRING in zweiter Ehe mit Katharina, 2) einer Tochter des Ratsherrn HinRICH Joris, dessen Wappen zu Häupten der Dargestellten, drei grüne, abwechselnd mit roten und silbernen Rosen belegte Kränze, 2 : I, in Gold zeigt, und als Helmzier einer grünen Kranz zwischen abwechselnd grün und gold geteilten Büffelhörnern. Das mütterliche Wappen dieser Seite, ein nach rechts stehender brauner Wiedehopf in Gold, Helmzier grüner Drache, habe ich hier nicht festzustellen vermocht.

Das werk trägt die volle Bezeichnung des Malers. Sie befindet sich links in der Höhe des Kopfes des Ratsherrn und war ursprünglich auf gelber Untermalung mit Gold aufgesetzt, innerhalb einer in gleicher Weise ausgeführten schildartigen Umrandung, wurde aber, wahrscheinlich bei einer um $185^{\circ}$ unternommenen Restaurierung, wobei auch die Aermel des ratsherrlichen Gewandes die erwähnte unschöne Uebermalung erhielten - zum Glück die einzige, ausser einigen geringfügigen Ausbesserungen - mit schwarzer Farbe überzogen. Mit der Zeit versank dadurch die Bezeichnung so in den schwarzen Hintergrund des Porträts, dass nur einem glücklichen Zufall - als die Tafel einmal in eine intensive seitliche Beleuchtung gerückt wurde - die Wiederauffindung zu danken ist. Mochte dem Restaurator aus ästhetischen Gründen die Inschrift in der Nähe des Kopfes missfallen, dass er sie übermalte, oder war es ihr ruinöser Zustand, jedenfalls ging er bei der Uebermalung sehr unachtsam zu Werke, auch verstand er vielleicht nicht genügend Latein, um sie getreu wiederzugeben. Sie lautet jetzt:

$$
\text { MO-JACOBUS TRAJVCTSIS-- v h }
$$

Oder ursprünglich :

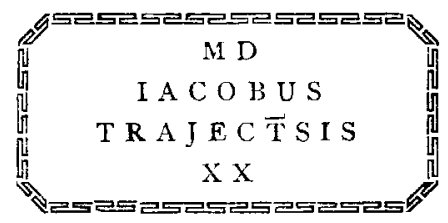

1) Seid Epitaph in der Marienkirche zu Lübeck.

2) De portretten van een zoon van dit echtpaar: THOMas Kerckrinck 1565 aet. 34 en zijne vrouw LUCRETIA JANSDr. VAN BASSEN, I565 aet. 20, beide levensgroote kniestukken met opschrift en wapen bevinden zich $b i j$ den heer N. M. C. Holland, te 's-Gravenhage. Het zijn goede werken van Amsterdninsche portrelteerkunst. Zie over de Amsterdamsche nakomelingen van dit geslacht: EliAs Vroedschap van Amsterdam II blz. 9 I4 v.v. C. H. de G. 
Es ist also in dem Worte Trajectensis das $\mathrm{E}$ in ein $\mathrm{U}$ verwandelt und der die Abreviatur für die Buchstaben $E N$ ausdrückende Strich über dem folgenden $T$ fortgelassen. Die Buchstaben MO über dem Namen werden M D = 1500 zu lesen sein und die völlig verunstalteten Zeichen unter dem Namen für $\mathrm{XX}=20$, was für die Zeit der Herstellung das Jahr 1520 ergäbe. Diese Jahreszahl stimmt auch mit der auf der zierlichen Schliesse angebrachten überein, die als Rest der ehemaligen Umrahmung erhalten ist. Diese ist mit Beibehaltung der alten Formen völlig erneuert und vergoldet, während sie vermutlich ehemals rot mit goldenen Sternen besetzt gewesen sein wird.

Das zierliche Werk ist, mit Ausnahme der erwähnten Uebermalungen, durchweg vortrefflich erhalten, selbst die zarten Lasuren auf dem Bilde der Jungfrau stehen in ursprünglicher Frische da. Die Farbe ist leuchtend, der Vortrag verschmolzen, die Ausführung eine durchweg sorgfältige.

Von den Arbeiten des Meisters waren bis jetzt 1) vier Porträts bekannt, von denen das weibliche Bildnis in der ehemaligen Galerie Weber-Hamburg in seiner auffallenden Uebereinstimmung mit dem Rigaer weiblichen Portrait, das 1520 entstanden ist, als ebenfalls in diese Zeit fallend angesehen werden kann. Der Rigaer Altar und das Hamburger Bild würden somit als die ältesten in Deutschland entstandenen Werke des Meisters zu gelten haben. Dem Jahre I523 gehört das Brustbild eines weissbärtigen Mannes im Kaiser Friedrich-Museum in Berlin an. Er trägt einen schwarzen mit breiten, gelbbesäumten Streifen eingefassten Mantel; den Kopf bedeckt ein runder schwarzer Hut, unter dem eine leuchtend rote Kappe zum Vorschein kommt. Den Hintergrund bildet eine felsige, von breiten Wasserläufen durchzogene Landschaft (Abb. im Katalog d. Kais. Friedr. Mus.) - Vom Jahre ${ }_{1} 5_{24}$ ist das Brustbild eines jungen Mannes datiert, der beim Schreiben eines Briefes nachdenkend nach oben blickt. Ehemals in der Sammlung des Freiherrn Alexander v. Minutoli-Waldeck auf Schloss Fridersdorf bei Greifenberg in Schlesien, jetzt in der Sammlung des Herrn Dr: FREund in Berlin. (Beschrieben v. HENRY THODE in d. Zeitschr. f. bild. Kunst i 886 S. $3^{2}$ ). Das vierte Bild im Nationalmuseum in Stockholm zeigt einen Mann mit übereinandergelegten Händen vor einem Tische mit den Resten eines Mahles. Es ist undatiert. Wie der Rigaer Hausaltar tragen auch die übrigen Bilder, mit Ausnahme des im Kaiser FrIEDRICH-Museum befindlichen, Wappen und zwar das ehemalige Hamburger Portrait einen gespaltenen Schild,

1) Sinds het overlijden van den schrijver zijn er verscheiden andere aan het licht gekomen, o.a. een in de verzameling Stokvis te 's-Gravenhage, drie op het slot Herdringen in Westfalen (afgebeeld in de Bau- und Kunstdenkmäler van Westfalen) en een in de veiling C. Hoogendijk, Mei rgi 2 Nr. 86 (afgeb. in den cat. 1532 aet. 30 gemerkt. Van het schilderij der verz. Weber is een beter exemplaar als geschenk Acconati Visconti in het Louvre gekomen. 
vorn einen Flügel, hinten eine Weinrebe. Einen silbernen Flügel in Schwarz innerhalb mit Schwarz und Silber gestickter Schildbordüre, vorne eine Weinrebe in Gold; Helmzier: Weinrebe zwischen silbernem mit schwarzen Balken belegten Flug, führte die Familie Fluwerk in Lüneburg. (cf. Siebmacher: Bürgerliches Wappenbuch V, 5. S. 75 Taf. 86). Beim Vergleich der Frauentrachten auf dem Rigaer und dem Hamburger Bilde wird man annehmen dürfen, dass auch dieses in Lübeck entstanden ist. - Auf dem Bilde im Besitz des Herrn Dr. Freund sieht man zwei Wappen: Auf dem vorderen einen roten Greif in Silber, auf dem anderen einen aufgezäumten Pferdekopf in Gold. Das erste lässt sich nach Siebmacher als das der Lübecker Familie BRUNS, das andere als das der Familie Swarte (Schwarze) in Stralsund ansprechen. - Das Stockholmer Bild hat ein Wappen mit einem Schiff, das in verschiedenen Formen - von der mittelalterlichen Kogge bis zum Vollschiff sowohl von Lübecker wie von Hamburger Familien geführt worden ist.

Was wir über JACOBUS van Utrecht wissen, ist wenig. Ein Meister seines Namens wird ${ }^{5} 56$ in die St. Lucasgilde zu Antwerpen aufgenommen und ist dort bis $15^{12}$ ansässig. Seine künstlerische Tätigkeit lässt sich vorläufig nur von I 520 bis ${ }^{2} 5^{24}$ an in Deutschland entstandenen Gemälden verfolgen. Er ist ein Zeitgenosse von JAN GOSSAERT und von JACOB CORNELISZ van Amsterdam, dem er sehr nahe steht. An diesen erinnert er besonders in den Szenen aus dem Landleben und in deren liebevoller Rehandlung wie sie auch auf dessen Altärchen im Kaiser FrIEDRICH-Museum erscheinen. Weder seine Madonna noch der Jesusknabe sind ideale schönheiten; auch darin erinnert er an JACOB CoRnelisz. Was man als italienischen Einfluss in dem Madonnenbilde des Rigaer Altars bezeichnen kann, mag auf die allgemeine Kunstrichtung der niederländischen Malerei seiner Zeit zu setzen sein. Dass er seine Studien in Deutschland fortgesetzt und den Einfluss Dürers erfahren hat, erscheint zweifellos. Einen längeren Aufenthalt in Lübeck vielleicht auch in Hamburg, oder in anderen benachbarten Hansestädten bezeugen die bis jetzt bekannt gewordenen Werke seiner Hand. Allenfalls könnte der Tracht nach zu urteilen, das Berliner Bild in Süd- oder Mitteldeutschland entstanden sein. Vielleicht gelingt es der Lokalforschung weiteres Material über den Meister herbeizuschaffen.

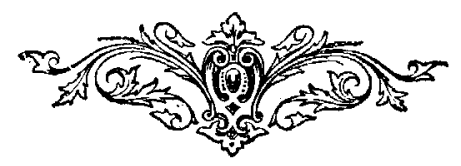

